\title{
Electronic Structure and Transport in Approximants of the Penrose Tiling
}

\author{
G. Trambly de Laissardière ${ }^{a}$, A. SzÁllás ${ }^{b}$ And D. MAYOU ${ }^{c}$ \\ ${ }^{a}$ Laboratoire de Physique Théorique et Modélisation, CNRS and Université de Cergy-Pontoise \\ F-95302 Cergy-Pontoise, France \\ ${ }^{b}$ Wigner Research Centre for Physics, P.O. Box 49, H-1525 Budapest, Hungary \\ ${ }^{c}$ Univ. Grenoble Alpes, CNRS, Institut NEEL, F-38042 Grenoble, France
}

\begin{abstract}
We present numerical calculations of electronic structure and transport in the Penrose approximants. The electronic structure of perfect approximants shows a spiky density of states and a tendency to localization that is more pronounced in the middle of the band. Near the band edges the behavior is more similar to that of free electrons. These calculations of band structure and in particular the band scaling suggest an anomalous quantum diffusion when compared to normal ballistic crystals. This is confirmed by a numerical calculation of quantum diffusion which shows a crossover from normal ballistic propagation at long times to anomalous, possibly insulator-like, behavior at short times. The time scale $t^{*}(E)$ for this crossover is computed for several approximants and is detailed. The consequences for electronic conductivity are discussed in the context of the relaxation time approximation. The metallic-like or non-metallic-like behavior of the conductivity is dictated by the comparison between the scattering time due to defects and the time scale $t^{*}(E)$.
\end{abstract}

DOI: 10.12693/APhysPolA.126.617

PACS: 71.23.Ft, 71.30.+h, 72.15.Rn, 71.23.An

\section{Introduction}

Since the discovery of Shechtman et al. [1] numerous experimental studies indicated that the conduction properties of several stable quasicrystals (AlCuFe, AlPdMn, AlPdRe...) are quite opposite to those of good metals [2-6]. It appears also that the medium range order, over one or a few nanometers, is the relevant length scale that determines conductivity. In particular, the role of transition elements enhancing localization has been often studied [7-12]. There is now strong evidence that these nonstandard properties result from a new type of breakdown of the semiclassical Bloch-Boltzmann theory of conduction [13-16]. On the other hand, the specific role of long range quasiperiodic order on transport properties is still an open question in spite of a large number of studies (see Refs. [17-34] and Refs. therein).

In this paper, we study "how electrons propagate" in approximants of the rhombic Penrose tiling P3 (PT in what follows). This tiling is one of the well-known quasiperiodic tilings that have been used to understand the influence of quasiperiodicity on electronic transport $[19,20,22,23,30,32,33]$. The main objective is to show that nonstandard conduction properties result from purely quantum effects due to quasiperiodicity that cannot be interpreted through the semiclassical theory of transport.

\section{Approximants of Penrose tiling}

To study electronic properties of PT, we consider a series of periodic approximants, called Taylor approximants, proposed by Duneau and Audier [35]. These approximants have defects as compared to the infinite per- fect tiling, but the relative number of defects becomes negligible as their size increases. They have been used to study the magnetic properties of PT $[36,37]$. Here we study electronic structure and quantum diffusion in three Taylor approximants, $T=3,4$, and 5 . Their rectangular cells $L_{x} \times L_{y}$ are $24.80 a \times 21.09 a, 40.12 a \times 34.13 a$, and $64.92 a \times 55.23 a$, respectively. $a$ is the tile edge length. They contain 644,1686 , and 4414 sites, respectively.

\section{Electronic structure}

We study a pure hopping Hamiltonian

$$
\hat{H}=\gamma \sum_{\langle i, j\rangle}|i\rangle\langle j|,
$$

where $i$ indexes $s$ orbitals $|i\rangle$ located on all vertexes. For realistic order of magnitude of the model one can choose the strength of the hopping between orbitals $\gamma=1 \mathrm{eV}$. Indices $i, j$ label the nearest neighbors at tile edge distance $a$. The properties of this model depend only on the topology of the tiling. The electronic eigenstates $|n \boldsymbol{k}\rangle$, with wave vector $\boldsymbol{k}$ and energy $E_{\boldsymbol{n}}(\boldsymbol{k})$, are computed by diagonalization in the reciprocal space for a number $N_{k}$ of vectors $\boldsymbol{k}$ in the first Brillouin zone. The density of states (DOS), $n(E)$, is calculated by

$$
n(E)=\langle\delta(E-\hat{H})\rangle_{E_{n}=E},
$$

where $\langle\ldots\rangle_{E_{n}=E}$ is the average on states with energy $E$. It is obtained by taking the eigenstates for each $\boldsymbol{k}$ vector with energy $E_{n}(\boldsymbol{k})$ such that $E-\delta E / 2<E_{n}(\boldsymbol{k})<$ $E+\delta E / 2 . \delta E$ is the energy resolution of the calculation. When $N_{k}$ is too small, the calculated quantities are sensitive to $N_{k}$. Therefore $N_{k}$ is increased until the results do not depend significantly on $N_{k}$. We use $\delta E=0.01 \mathrm{eV}$, 
$N_{k}=144^{2}, 96^{2}$, and $48^{2}$ for Taylor approximants $T=3$, 4 , and 5 , respectively.

\subsection{Density of states}

The density of states is shown in Fig. 1a. As expected in $[17,28,29]$, it is symmetric with respect to $E=0$. The main characteristic of these DOS are similar to that obtained by Zijlstra [28, 29], for other family of the Penrose approximants. At $E=0$ a strictly localized state is obtained [17,38]. A gap is found for energy $|E| \lesssim 0.13 \mathrm{eV}$ and a small gap with a width less than $0.01 \mathrm{eV}$ seems to be at $|E| \approx 2.7 \mathrm{eV}[28,29]$. Other fine gaps could be present at $|E| \approx 0.3,0.5,1.7 \mathrm{eV}(\ldots)$ but our energy resolution cannot obtain them. The DOS is more spiky at the center of the band $(|E|<2)$ and smooth near the band edges $(|E|>2)$.

\subsection{Participation ratio}

In order to quantify this localization phenomenon, we compute the average participation ratio defined by:

$$
p(E)=\left\langle\left(N \sum_{i=1}^{N}|\langle i \mid n \boldsymbol{k}\rangle|^{4}\right)^{-1}\right\rangle_{E_{n}=E},
$$

where $i$ indexes orbitals in a unit cell and $N$ is the number of orbitals in this unit cell. For completely delocalized eigenstates $p$ is equal to 1 . On the other hand, states localized on one site have a small $p$ value: $p=1 / N$. Figure 1b shows clearly a stronger localization of electronic states for larger approximants.

\subsection{Band scaling}

The average Boltzmann velocity along the $x$ direction is computed by

$$
V_{\mathrm{B}}(E)=\sqrt{\left\langle\left|\left\langle n \boldsymbol{k}\left|\hat{V}_{x}\right| n \boldsymbol{k}\right\rangle\right|^{2}\right\rangle_{E_{n}=E}},
$$

where the velocity operator along the $x$ direction is $\hat{V}_{x}=[\hat{X}, \hat{H}] /(\mathrm{i} \hbar)$, with $\hat{X}$ the position operator. $V_{\mathrm{B}}$ is the average intra-band velocity,

$$
V_{\mathrm{B}}(E)=\frac{1}{\hbar}\left\langle\frac{\partial E_{n}(\boldsymbol{k})}{\partial k_{x}}\right\rangle_{E_{n}=E} .
$$

Figure 1c shows a smaller velocity at the center of the band $(|E|<2)$. When the size of the approximant increases, $V_{\mathrm{B}}$ decreases as expected from band scaling analysis [16, 19-21]. Typically the width $\Delta E$ of a band $E_{n}(\boldsymbol{k})$ varies in the $k_{x}$ direction like, $\Delta E \propto L_{x}^{-\Gamma}$, where $L_{x}$ is the length of the unit cell in the $x$ direction. The exponent $\Gamma$ depends on $E$ and the diffusion properties of the structure. For normal metallic crystals $\Gamma=1$, for disordered metallic alloys the electronic states are diffusive and $\Gamma=2$. From Eq. (5), the Boltzmann velocity should satisfy that $V_{\mathrm{B}} \propto L_{x}^{1-\Gamma}$. Figure $1 \mathrm{~d}$ shows $V_{\mathrm{B}} L_{x}^{\Gamma-1}$ versus energy $E$. For $\Gamma \approx 2$ the value of $V_{\mathrm{B}}(E) L_{x}^{\Gamma-1}$ are rather similar for the three approximants at the center of the band $(|E|<2)$. For $2<|E|<3.5$, it seems that $\Gamma \approx 1.5$, and near the band edges, $|E|>3.5$, states are almost ballistic $\Gamma \approx 1$.
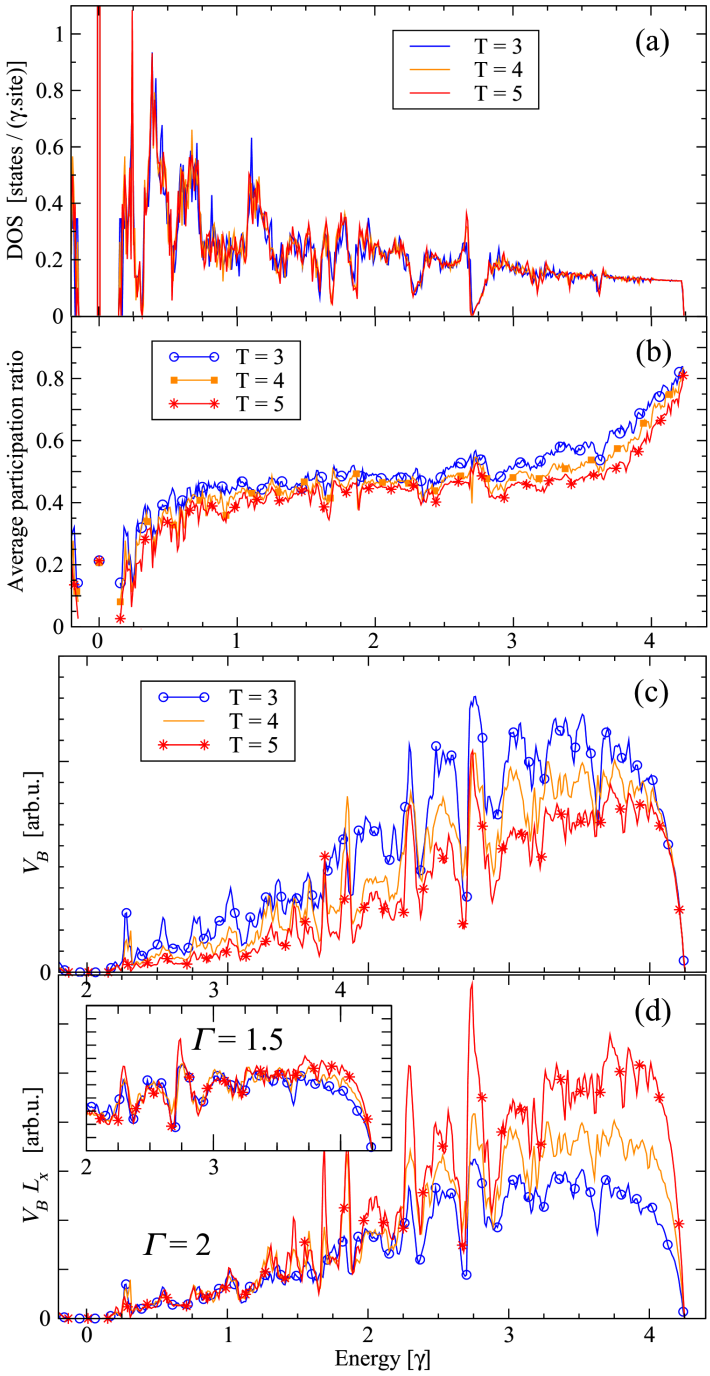

Fig. 1. Electronic structure in Penrose approximants. (a) Total density of states (DOS) $n(E)$. DOS is symmetric with respect to $E=0$. (b) Average participation ratio $p(E)$. (c) Average Boltzmann velocity $V_{\mathrm{B}}(E)$ along the $x$ direction, (d) $V_{\mathrm{B}}(E) \times L_{x}^{\Gamma-1}$ versus energy $E$ for $\Gamma=2$ (inset: $\Gamma=1.5$ ).

\section{Electronic transport}

\subsection{Quantum diffusion}

The band scaling has a direct consequence for the wave propagation in the medium. The mean spreading, $L_{\mathrm{wp}}(t)$ of a wave packet is neither ballistic (i.e. proportional to time $t$ ) as in perfect crystals nor diffusive (i.e. $\left.L_{\mathrm{wp}}(t) \propto \sqrt{t}\right)$ as in disordered metals. In general at large $t$ :

$$
L_{\mathrm{wp}}(E, t) \propto t^{\beta(E)} .
$$

The value of the exponent $\beta$ in quasicrystals (or in approximants with size cell $L_{x}$ going to infinity) can be related to $\Gamma$ in finite approximants by $\beta=1 / \Gamma[16]$. Thus our results on approximants show that states in $\mathrm{PT}$ are diffusive $(\beta \approx 0.5)$ at the center of the band $(|E|<2)$, 
super-diffusive $(0.5<\beta<1)$ for $2<|E|<3.5$, and almost ballistic $(\beta \approx 1)$ near the band edges $(|E|>3.5)$.

It is possible to go beyond these qualitative arguments by defining in an exact manner the quantum diffusion as we show now. The average square spreading of states of energy $E$ at time $t$ along the $x$ direction, is defined as:

$$
X^{2}(E, t)=\left\langle(\hat{X}(t)-\hat{X}(0))^{2}\right\rangle_{E},
$$

with $\hat{X}(t)$ - the Heisenberg representation of the operator. It can be shown that $\hat{X}$ is the sum of two terms $[13,15]$ :

$$
X^{2}(E, t)=V_{\mathrm{B}}^{2}(E) t^{2}+X_{\mathrm{nB}}^{2}(E, t) .
$$

The first term, $X_{\mathrm{B}}=V_{\mathrm{B}}^{2}(E) t^{2}$, is the ballistic (intra-band) contribution at energy $E$. The semiclassical model of the Bloch-Boltzmann transport theory amounts to taking into account only this first term. The second term (inter-band contributions) $X_{\mathrm{nB}}^{2}(E, t)$ is a non-Boltzmann contribution. It is due to the non-diagonal elements of the velocity operator and describes a spreading of the wave function.
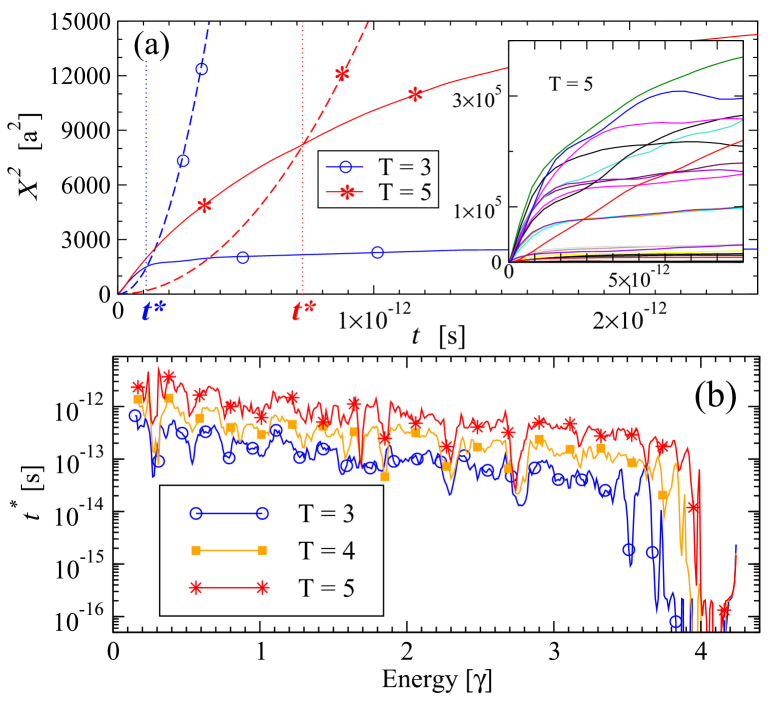

Fig. 2. (a) Average square spreading versus time $t$ at $E=1.0 \mathrm{eV}$ in perfect Penrose approximants $T=3$ and 5: (dashed line) Boltzmann $X_{\mathrm{B}}^{2}$ and (line) non-Boltzmann $X_{\mathrm{nB}}^{2}$ (inset: $X_{\mathrm{nB}}^{2}$ versus time $t$ at different energies in approximant $T=5$ ). (b) Time $t^{*}$ in perfect Penrose approximants (see text).

One defines the time $t^{*}(E)$ for which $X_{\mathrm{B}}^{2}=X_{\mathrm{nB}}^{2}$ at energy $E$ (Fig. 2a). For long times, $t>t^{*}$, the ballistic semiclassical contribution dominates the quantum diffusion but for short times, $t<t^{*}$, the non-ballistic contribution dominates ("low velocity regime" [13]). Therefore $t^{*}(E)$ is important time scales for any approximant. On time scales larger than $t^{*}(E)$ the approximant behaves like a normal metal whereas on smaller time scales the approximant may behave quite differently and, in particular, may show insulator-like behavior (see below). When the size of the approximants increases, the charac- teristic time limit $t^{*}$ of the crossover between ballistic and non-ballistic behavior increases (Fig. 2b). Similar results has been found in approximants of octagonal tiling [34]. From $a b$ initio electronic structure calculation and in realistic approximants $\alpha$-AlMnSi [13], 1/1 AlCuFe [14] and in the complex metallic hexagonal phase $\lambda$-AlMn [15] it has been shown that the order of magnitude of $t^{*}(E)$ is about $10^{-14}$ or $10^{-13} \mathrm{~s}$.

$X_{\mathrm{nB}}^{2}(E, t)$ oscillates and is bounded by $L_{\mathrm{nB}}(E)^{2}$, which depends on the energy $E[15]$. From numerical calculations (Fig. 2a), it is found that for many energies $E$, $X_{\mathrm{nB}}^{2}(E, t)$ reaches rapidly its maximum limit $L_{\mathrm{nB}}(E)^{2}$ and one can assume, $X_{\mathrm{nB}}(E, t)^{2} \approx L_{\mathrm{nB}}(E)^{2}$ for large $t$, and $t^{*} \approx L_{\mathrm{nB}} / V_{\mathrm{B}}$.

\subsection{Conductivity in the relaxation time approximation}

In the relaxation time approximation (RTA) $[13,16,25]$ the role of phonons and static defects are taken into account through a scattering time $\tau$. $\tau$ decreases when temperature $T$ increases and when the number of static defects increases. The scattering time estimates in quasicrystals and approximants from transport measurements at low temperature $(4 \mathrm{~K})[2,3]$ is about a few $10^{-14} \mathrm{~s}$ or even more. That is close to the time limit $t^{*}$ between the Boltzmann and non-Boltzmann behavior (see previous section). Therefore the non-Boltzmann behavior could play a crucial role in the conductivity.

At zero temperature, the static conductivity is given by the Einstein formula,

$$
\sigma=e^{2} n\left(E_{\mathrm{F}}\right) D\left(E_{\mathrm{F}}\right),
$$

where $E_{\mathrm{F}}$ is the Fermi energy and $D$, the diffusivity which is the sum of a Boltzmann and non-Boltzmann terms [13]:

$D\left(E_{\mathrm{F}}\right)=D_{\mathrm{B}}+D_{\mathrm{nB}}\left(E_{\mathrm{F}}\right)$, with $D_{\mathrm{B}}=V_{\mathrm{B}}^{2}\left(E_{\mathrm{F}}\right) \tau$. (10) $D_{\mathrm{nB}}\left(E_{\mathrm{F}}\right)$ is calculated numerically from eigenstates (see $[15,16])$.

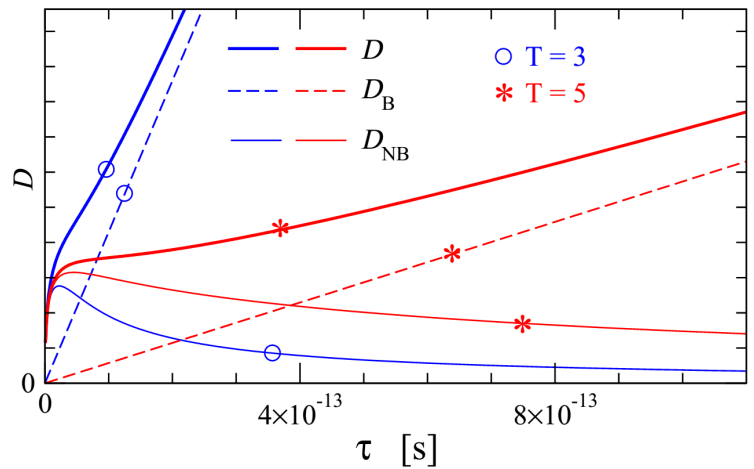

Fig. 3. Diffusivity $D, D=D_{\mathrm{B}}+D_{\mathrm{nB}}$, in relaxation time approximation, versus scattering time $\tau$, at $E_{\mathrm{F}}=$ $1.0 \mathrm{eV}$ in the Penrose approximants $T=3$ and 5 .

Figure 3 shows the diffusivity in approximants $T=3$ and 5 for $E_{\mathrm{F}}=1 \mathrm{eV}$. At very low $\tau, \tau \lesssim 10^{-15} \mathrm{~s}$, diffusivity is always ballistic, for larger $\tau$ values up to $\tau \approx t^{*}$ 
the non-Boltzmann terms dominate, and for $\tau \gg t^{*}$ periodicity of approximants induces ballistic diffusivity. The intermediate zone, with a non-metallic (non-ballistic) behavior due to structure, is more important in the largest approximant, and it corresponds to realistic values of scattering time.

\section{Conclusion}

To summarize, we have presented a numerical study of electronic structure and quantum diffusion for a pure hopping Hamiltonian in approximants of the Penrose tiling containing 644,1686 , and 4414 sites in a unit cell. When the size of the unit cell of the approximant increases the usual Boltzmann term for quantum diffusion (ballistic term) decreases rapidly and non-Boltzmann terms become essential to understand transport properties. These non-Boltzmann terms can have "insulator-like" behavior, suggesting that in larger approximants, "insulator-like" states, due to long range quasiperiodic order, could exist. Calculations in larger approximants are in progress.

\section{Acknowledgments}

We thank A. Jagannathan for fruitfull discussions. The computations were performed at the Centre de Calcul of the Université de Cergy-Pontoise. We thank Y. Costes and D. Domergue for computing assistance.

\section{References}

[1] D. Shechtman, J. Blech, D. Gratias, J.W. Cahn, Phys. Rev. Lett. 53, 1951 (1984).

[2] S.J. Poon, Adv. Phys. 41, 303 (1992).

[3] C. Berger, E. Belin, D. Mayou, Ann. Chim. Mater (Paris) 18, 485 (1993).

[4] E. Belin, D. Mayou, Phys. Scr. T 49A, 356 (1993).

[5] C. Berger, in: Lectures on Quasicrystals, Eds. F. Hippert, D. Gratias, Les Editions de Physique, Les Ulis 1994, p. 463.

[6] T. Grenet, in: Quasicrystals: Current Topics, Eds. E. Belin-Ferré, C. Berger, M. Quiquandon, A. Sadoc, World Sci., Singapore 2000, p. 455.

[7] T. Fujiwara, Phys. Rev. B 40, 942 (1989).

[8] T. Fujiwara, S. Yamamoto, G. Trambly de Laissardière, Phys. Rev. Lett. 71, 4166 (1993).

[9] M. Krajčí, M. Windisch, J. Hafner, G. Kresse, M. Mihalkovič, Phys. Rev. B 51, 17355 (1995).

[10] G. Trambly de Laissardière, D. Mayou, Phys. Rev. B 55, 2890 (1997).

[11] G. Trambly de Laissardière, S. Roche, D. Mayou, Mater. Sci. Eng. A 226-228, 986 (1997).
[12] G. Trambly de Laissardière, D. Nguyen Manh, D. Mayou, Prog. Mater. Sci. 50, 679 (2005).

[13] G. Trambly de Laissardière, J.P. Julien, D. Mayou, Phys. Rev. Lett. 97, 026601 (2006).

[14] G. Trambly de Laissardière, J.P. Julien, D. Mayou, Philos. Mag. 88, 2131 (2008).

[15] G. Trambly de Laissardière, Mayou, C.R. Physique 15, 70 (2014)

[16] D. Mayou, G. Trambly de Laissardière, in: Quasicrystals, series Handbook of Metal Physics, Eds. T. Fujiwara, Y. Ishii, Elsevier Science, Oxford 2008, Vol. 3, p. 209.

[17] M. Kohmoto, B. Sutherland, Phys. Rev. Lett. 56, 2740 (1986)

[18] T. Fujiwara, M. Kohmoto, T. Tokihiro, Phys. Rev. B 40, 7413 (1989).

[19] C. Sire, J. Bellissard, Europhys. Lett. 11, 439 (1990).

[20] B. Passaro, C. Sire, V.G. Benza, Phys. Rev. B 46, 13751 (1992).

[21] C. Sire, in Ref. [5], p. 505.

[22] A. Jagannathan, J. Phys. I (France) 4, 133 (1994).

[23] J.X. Zhong, R. Mosseri, J. Phys. I (France) 4, 1513 (1994).

[24] S. Roche, D. Mayou, Phys. Rev. Lett. 79, 2518 (1997); S. Roche, D. Mayou, Phys. Rev. B 60, 322 (1999).

[25] D. Mayou, Phys. Rev. Lett. 85, 1290 (2000).

[26] S. Yamamoto, T. Fujiwara, Phys. Rev. B 51, 8841 (1995).

[27] T. Fujiwara, T. Mitsui, S. Yamamoto, Phys. Rev. B 53, R2910 (1996).

[28] E.S. Zijlstra, T. Janssen, Phys. Rev. B 61, 3377 (2000).

[29] E.S. Zijlstra, T. Janssen, Mater. Sci. Eng. 294-296, 886 (2000).

[30] E.S. Zijlstra, Phys. Rev. B 66, 214202 (2002).

[31] F. Triozon, Julien Vidal, R. Mosseri, D. Mayou, Phys. Rev. B 65, R220202 (2002).

[32] E.S. Zijlstra, J. Non-Cryst. Solids 334, 126 (2004).

[33] A. Jagannathan, P. Piéchon, Philos. Mag. 87, 2389 (2007).

[34] G. Trambly, C. de Laissardière, D. Oguey, Mayou, Philos. Mag. 91, 2778 (2011).

[35] M. Duneau, M. Audier, in Ref. [5], p. 283.

[36] A. Jagannathan, A. Szallas, S. Wessel, M. Duneau, Phys. Rev. B 75, 212407 (2007).

[37] A. Szallas, A. Jagannathan, Phys. Rev. B 77, 104427 (2008).

[38] M. Arai, T. Tokihiro, T. Fujiwara, M. Kohmoto, Phys. Rev. B 38, 1621 (1988). 